information on the ones in current use. (As in the Bible, there is a 'begat' chapter with all sorts of details of family histories, carefully researched by Harris before memories fade and sources are forever forgotten.) Also included are descriptions of many useful biochemical techniques and methodologies for molecular biological work on such unicellular algae.

There are, too, good reviews of the sexuality of these versatile microbes, the feature which, above all else, led to their selection for detailed studies. Seventy years ago, the first direct confirmation of mendelian inheritance came from a study of Chlamydomonas, but for 30 years after that there were few publications on this subject (and many of those, regrettably, proved to be largely fictional). Only in the late $1940 \mathrm{~s}$ did a handful of biologists, seeking a single-celled plantlet with genetic potential, take up studies of the genus. Mostly we chose to work with one of two unrelated species, C. moewusii (also known as $C$. eugametos) and $C$. reinhardtii. Their sexual proclivities are sometimes melodramatic, or even furtive, but by now they have been elucidated fairly well and are under good laboratory control. Unfortunately $C$. moewusii, which lends itself best to studies of sexuality, suffers from a metabolic disability all too common among algae, an inability to grow heterotrophically in darkness, so in this species photosynthesis cannot be bypassed. Consequently $C$. reinhardtii, with its more versatile metabolic potentials, has been the species of choice.

If I have to find a bone to pick, it would be on this point. When you read in Harris's book something about Chlamydomonas, you can generally assume that it relates to $C$. reinhardtii unless otherwise specified. However, generalizations are dangerous in a 'genus' as diverse as this - what's true for Peter may not be applicable to Paul and doubtless examination of other species will reveal exceptions and surprises of all sorts.

I note that on p.175 the first equation, misquoted from Chiang et al., is incorrect, and that on p. $130 \mathrm{C}$. philotes is wrongly given as heterothallic. But I have discovered nothing of importance that's missing, and lots of fascinating details that I knew nothing about before. There are full references to almost 3,000 publications, and abundant tables, diagrams, photographs and other illustrations, with adequate scales. A good ten-page, three-column index is provided. It's all there, in a stout binding and a hard, waterproof cover. The book will inevitably need both

Ralph A. Lewin is in the Marine Biology Research Division, Scripps Institution of Oceanography, University of California San Diego, La Jolla, California 92093, USA.

\section{Far off and exceeding deep}

\section{Harry Shipman and Noel Hart}

Deep Time: The Journey of a Single Subatomic Particle from the Moment of Creation to the Death of the Universe and Beyond. By David Darling. Bantam, London/Delacorte, New York: 1989. Pp.192. £12.95, \$17.95.

From Quarks to the Cosmos: Tools of Discovery. By Leon M. Lederman and David N. Schramm. W.H. Freeman: 1989. Pp.242. £16.95, \$32.95.

How did the Universe begin? Where will it end? These questions have taken on a new, rich dimension in the 1980 s with the development of a connection between cosmology and particle physics. In producing these two books, Darling and Lederman and Schramm join the crowd of scientists and journalists writing on the subject for a 'popular' audience (whatever that is).

Deep Time is a 'prose poem' telling the story of a proton which journeys from the Big Bang to the end of cosmic evolution. Some episodes in this journey illustrate a different and entertaining way of describing astronomical phenomena. Our proton hero narrowly escapes being sucked into a black hole in the centre of a galaxy, and along the way there is a nice description of the current central dogma about quasar energy sources.

But any writer takes on a real challenge in writing a book-length 'poem'. Lewis Carroll pulled it off in Alice in Wonderland, but Darling overdoses the reader with phrases such as "the hot breath of genesis", "oases of order" and "the mighty fortress of the second law" (of thermodynamics). One of us (H. S.) found the language merely bothersome; the other (N. H.) found it virtually unreadable.

It is hard to draw distinctions between well-founded theory and scientific speculation in a poem. Darling doesn't bother. For instance, he implies that cosmic strings must be midwives that assist in the birth of galaxies. Yet we don't even know whether cosmic strings exist. Fiction comes in, too, with tales of Prospero, the tenth planet in the Solar System. What tenth planet? Inexpert readers are here being led into the land of the tabloids, where the difference between science, pseudoscience and science fiction is considered to be irrelevant. Such readers will also have to struggle with sentences that presume a more than passing familiarity with, for instance, antileptons and antibaryons.

Lederman and Schramm provide a more conventional treatment of cosmology and its interface with particle physics.
The pictures are outstanding and serve well to illustrate the sparse text, while Lederman's perspective as an experimental particle physicist shows through nicely. Many pages clearly and thoroughly, though ponderously, describe mammoth particle accelerators and the associated array of detectors. Most popular books on this topic let the stork deliver new particles, such as the omega-minuses, from the clear blue sky. The authors here give us the facts of life, describing the 50,000 pictures which had to be taken before the omega-minus appeared to confirm the symmetric view of nature based on the quark model.

Lederman and Schramm, however, use jargon injudiciously. The book's second sentence describes the control room of Fermilab's Tevatron, but those who don't know what or where the Tevatron is must wait until page 115 for a pleasant, fourpage description of it. And the beat goes on: in the first five pages the reader is sprayed with bullets of terminology: antiprotons, p-bars, accumulator storage rings, luminosity, deflection 'kicker' magnets, GeV, CDF, standard model, quarks, leptons and more. One-sentence definitions of some of these bits of scientific lingo are not enough for the nonspecialist. Several pages later the level plunges precipitously. Using a box, the authors patiently explain powers of ten, and they take ten pages of text and many gorgeous illustrations to show that atoms are made of electrons and protons. Both reviewers, one of us a scientist and one a non-scientist, found the text to be like a rollercoaster ride and just as disorientating.

There is a plethora of cosmology books on the market already, including, of course, Hawking's blockbuster $A$ Brief History of Time. Why write or publish so many of them? That's a question the book-buying public should be asking.

Harry Shipman is in the Department of Physics and Astronomy, and Noel Hart the Department of Foreign Languages and Literatures, University of Delaware, Newark, Delaware 19716. USA.

- An English translation of Paolo Maffei's L'Universo nel tempo is now available; The Universe in Time, published by MIT Press, addresses issues not covered in Maffei's first book, Beyond the Moon. The Universe in Time is structured as a voyage in time, looking at the life cycle of the stars, planetary systems and eventually geological and ecological evolution. Price \$29.95, £26.95.

- Also recently translated, from the French, is Jean Heidmann's Cosmic Odyssey. The book was written to "give a description of the fabulous richness of the cosmos". It explores the relativistic universe, the quantum universe and the inflationary universe. Cosmic Odyssey is published by Cambridge University Press, price $£ 11.95, \$ 19.95$. 